\title{
A Novel Orthogonal Minimum Correlation Spreading Code in CDMA System
}

\author{
Shibashis Pradhan ${ }^{\mathrm{a}^{*}}$, Sudipta Chattopadhyay ${ }^{\mathrm{b}}$, Sujatarani Raut ${ }^{\mathrm{c}}$ \\ ${ }^{a}$ Department of ECE, Centurion University of Technology and Management,Paralakhemundi-761211, India \\ ${ }^{b}$ Department of Electronics and Telecommunication Engineering Jadavpur University, Kolkata-700032,India \\ ${ }^{c}$ Department of Electronics PN autonomous college, Khurda-752055, India
}

\begin{abstract}
Code Division Multiple Access (CDMA) is a technique in which transmission of information takes place simultaneously over the same available channel bandwidth. CDMA systems make use of spread spectrum (SS) technique for transmission of information by employing spreading codes. Each user is assigned with a unique spreading code which acts as a signature code for that individual user. The CDMA system experiences Multiple Access Interference (MAI) and Inter Symbol Interference (ISI) because of the non-orthogonality of the spreading codes. Both the MAI and ISI are the functions of auto-correlation and cross-correlation values of the spreading code respectively. In this paper, a novel orthogonal spreading code called "Orthogonal Minimum Correlation Spreading Code" (OMCSC) has been proposed which can serve a large number of users and is simultaneously expected to reduce the effect of MAI and ISI. Moreover, the Bit Error Rate (BER) performance of the proposed code has been compared with the existing codes using Additive White Gaussian Noise (AWGN) channel under multi-user scenario.
\end{abstract}

Index Terms: BER, CDMA, MAI, ISI, OMCSC, AWGN.

(C) 2014 Published by MECS Publisher. Selection and/or peer review under responsibility of the Research Association of Modern Education and Computer Science

\section{Introduction}

The performance of CDMA based wireless systems is largely based on the characteristics of user specific spreading codes [1]. These codes spread the original information signal to a large bandwidth and also provide unique identity to the various channels originating from base station as well as mobile station. At the receiver side, the original information signal is recovered by correlating the received signal with the synchronized replica of the spreading code [2-3]. Thus spreading codes play an important role in CDMA system to spread the user signals and distinguish among different users. Among the different spreading codes, Walsh code has proved its existence as a popular orthogonal code. On the other side Pseudo-Noise sequence (PN), Gold code

* Corresponding author. Tel.: +919778027532

E-mail address: shibashispradhan@ gmail.com 
and Kasami codes are found to be popular non-orthogonal binary spreading codes.

Non-orthogonality between spreading codes in CDMA system users causes MAI which consequently restricts the capacity of CDMA systems. Many strategies have been proposed to moderate the effect of MAI, for example, multiuser detection can be employed to use multiple user information for better detection of each user signal rather than using single conventional detection [4]. Here the conventional DS-CDMA detector follows a single user detection strategy in which each user is detected separately without regard for the other users. So a better strategy is multiuser detection, where information about multiple users is used to improve detection of each individual user.

Sarwate D. V. \& Pursley [5] presented a survey and provided several results on periodic and aperiodic crosscorrelation functions for pairs of m-sequences and for pairs of related (but not maximal-length) binary shift register sequences. The relation between the cross-correlation and autocorrelation function has also been included for complex-valued sequences. Multi-value CDMA spreading codes can be designed by means of a pair of mirror multi-rate filter banks based on some optimizing criterion [6]. It explains that there exists a theoretical bound in the performance of its circular correlation property, which is given by an explicit expression. Based on this analysis, a criterion of maximizing entropy is proposed to design such codes. A computer simulation result suggests that the resulting codes outperform the conventional binary balance Gold codes for an asynchronous CDMA system.

The problem of detecting the Optimal Spreading Codes (OSC) in a given code set for a Direct SequenceCode Division Multiple Access (DS-CDMA) system that employs de-spreading sequences weighted by adjustable chip waveforms has been described in [7]. Due to computational complexity required to detect the OSC in a code set, a simple and efficient strategy has been introduced. Analysis of numerical results show that the proposed strategy can successfully distinguish the spreading codes being most suitable references for a given code set in which the candidate spreading codes have equal cross correlation properties.

$\mathrm{Wu}$ and Nassar et al. [8] proposed a set of novel complex spreading codes called Carrier Interferometry (CI) codes and described how these novel orthogonal spreading codes achieved cross-correlations independent of the phase offsets between different paths after transmission over a multi-path fading channel. This improved cross-correlation property relative to Walsh codes leads to higher Signal to Interference Ratio (SIR) in the DSCDMA RAKE receiver, and, as a direct result, better performance in terms of probability of error had achieved.

Minimum auto correlation codes have been proposed in order to minimize the average magnitude of auto correlation with impulsive peak between spreading codes thereby minimizing the effect of ISI. It is shown that these codes have better average magnitude of auto correlation than Hadamard codes [9]. For example, for codes of lengths 8 and 16 the achievement in gain was $408 \%$ and 530\% respectively at one shift. The generation of minimum cross correlation spreading codes has been suggested in [10] in order to minimize the magnitude of cross correlation between different spreading codes. The average magnitude of cross correlation of the proposed code has been compared with that of Hadamard and Gold codes, and a noticeable enhancement over Hadamard and Gold codes has been achieved. In [11], minimum correlation spreading codes are presented in order to minimize the magnitude of auto correlation and cross correlation between spreading codes other than zero shift. The disadvantage of the work described in [9]-[11] is that each of them produces $\mathrm{N}-1$ number of spreading codes for a $\mathrm{N}$ length sequence which is less than Walsh code.

Generating orthogonal code sets of sequences with good correlation properties was developed by Donelan and Farrell in [12]. In this paper, an orthogonal code set has been defined as a collection of $\mathrm{N}$ number of sequences, each of length $\mathrm{N}$, which are mutually orthogonal. For $\mathrm{N}$-length code $(\mathrm{N}-1)$ the number of such code sets has been generated, each containing $\mathrm{N}$ number of orthogonal codes. Finally, $\mathrm{N} \times(\mathrm{N}-1)$ number of Orthogonal Gold codes have been produced. The peak cross-correlation values between the generated codes for various set sizes have also been measured. The measurement results indicate that the peak cross-correlation value improves for sets of size 32 and above, as the maximum value is less than half of the sequence.

In order to generate a new family of orthogonal code sets that can be employed as a spreading sequence in a DS-CDMA communication system, a small set of Kasami sequence has been utilized [13].The algorithm described in this paper is capable of providing more number of distinct codes than the popularly used Walsh 
code in CDMA system. The BER performance of the proposed code along with different orthogonal codes has been studied under multiuser scenario.

New sets of Walsh-like nonlinear phase orthogonal codes for synchronous and asynchronous CDMA communication system has been proposed in [14]. It has been directed that the proposed code outperforms Walsh code and their performances closely match with the nearly orthogonal Gold codes in AWGN channel with more number of codes than Walsh code. It has also been mentioned that the performances of all the binary codes are comparable to each other in Rayleigh flat-fading channels.

In this paper, a novel orthogonal minimum correlation spreading code has been proposed. The performance of the proposed code has been studied and compared with other existing codes in terms of various attributes, like number of generated codes, cross correlation and auto correlation values. To study the BER performance of the generated code, SIMULINK based downlink CDMA system model has been used. This paper is structured as follows: section 2 discusses about the various factors affecting the choice of spreading code; section 3 includes the mathematical background of spreading codes; the proposed algorithm has been presented in section 4; section 5 incorporates simulation results and its analysis; section 6 finally places some concluding remarks on this work and also some future scope of this work.

\section{Factor Affecting the Choice of Spreading Code}

There are many factors affecting the choice of spreading code namely cross correlation, auto correlation, length of code set and peak to average power ratio. However, in this paper correlation property is discussed in detail. Auto correlation [19] is a measure of the similarity between a code c (n) and its time shifted version.

Mathematically it is defined as:

$$
r_{\mathrm{cc}}(l)=\sum_{\mathrm{n}=-\infty}^{\infty} c(\mathrm{n}) * c(n-l) \quad \text { where }, l=0, \pm 1, \pm 2 \cdots
$$

Auto correlation function must be impulsive peak at zero time shifts and minimum values at all other shifts. It is essential at receiver side to differentiate the desired user from other users producing MAI. Thus, spreading code should be generated such that it gives maximum autocorrelation value at zero shifts and minimum other than at zero shifts. For example, Pseudo-Noise (PN) sequence generated using Linear Feedback Shift Register (LFSR) possess ideal impulsive auto correlation characteristic. But due to non orthogonality of the PN code its cross correlation property is not suitable for CDMA system. So spreading code for CDMA system should be generated in such a way that its Auto Correlation Function (ACF) must be impulsive in nature while maintaining minimum Cross Correlation Function (CCF) among them.

Cross correlation [19]-[20] is a measure of similarity of two waveforms as a function of time lag applied to one of them. In order to appreciate the relationship between MAI and cross correlation properties between spreading codes, let's investigate a simple CDMA system. Assume there are N synchronous users, the channel is single-path, users transmit at unity amplitude, and there is no phase shift between the signals. The uplink baseband received signal at any particular bit interval can be written as [4]

$$
r(t)=\sum_{i=1}^{N} h_{i} d_{i} s_{i}(t)+n(t)
$$

Where, $\mathrm{N}$ is no of users, si(t), di, hi, are spreading code, transmitted data bit and gain of ith user respectively in the CDMA system, and $n(t)$ represent system noise. By using correlator the output of ith user can be represented by equation given below 


$$
y_{i}=h_{i} d_{i}+\int n(t) s_{i}(t) d t \sum_{k=1, k \neq 1}^{N} h_{k} d_{k} \int s_{k}(t) s_{i}(t) d t
$$

It is obvious that yi is composed of three components: desired signal hi.di, noise effect, $\int \mathrm{n}(\mathrm{t}) \mathrm{s}_{\mathrm{i}}(\mathrm{t}) \mathrm{dt}$ and MAI for the ith user, $\sum_{k=1, k \neq 1}^{N} h_{k} d_{k} \int s_{k}(t) s_{i}(t) d t$. If cross correlation relation between spreading code of ith user and all other users, $\int \mathrm{s}_{\mathrm{k}}(\mathrm{t}) \mathrm{s}_{\mathrm{i}}(\mathrm{t}) \mathrm{dt}$ is zero then the ith user will not have a MAI term in yi. But in multiuser system MAI quantity must be minimized as it is not possible to generate codes with zero cross correlation value.

\section{Mathematical Background of Spreading Code}

\subsection{Walsh-Hadamard Codes}

Walsh Hadamard code generates orthogonal spreading codes for multicarrier CDMA system. Orthogonal codes avoid Multiple Access Interference under perfect synchronization. These functions are generated by [3] mapping codeword rows of a special square matrix called Hadamard matrix. So N length Walsh code can offer $\mathrm{N}$ number of codes which can assist maximum $\mathrm{N}$ number of CDMA users, and that can be generated by a standard repetative procedure:

$$
W_{l}=[0], \quad \mathrm{W}_{2}=\left[\begin{array}{ll}
0 & 0 \\
0 & 1
\end{array}\right], \quad W_{4}=\left[\begin{array}{llll}
0 & 0 & 0 & 0 \\
0 & 1 & 0 & 1 \\
0 & 0 & 1 & 1 \\
0 & 1 & 1 & 0
\end{array}\right] \text { and } W_{2 N}=\left[\begin{array}{ll}
W_{N} & W_{N} \\
W_{N} & W_{N}
\end{array}\right]
$$

Here Walsh matrix contain first row of all zeros and the next rows have equal number of ones and zeros. $\mathrm{N}$ is a power of 2 and over score implies the binary complement of corresponding bits in the matrix. Each row of the matrix represent a Walsh code by mapping 0 to 1 and 1 to -1 . So these generated codes are orthogonal and between any pair cross-correlation value is zero.

\subsection{Gold Codes}

Gold sequence can generate huge number of codes just by employing two simple functions shift and module2 addition. The sequence is choosen from set of codes whose cross correlation between the code is uniform and bounded [15] [16] [17]. Actually, the entire set of these codes are generated by modifying orginal Gold codes generated by preferred pair of maximal PN sequences. The family of orginal Gold codes is defined by $\left\{a, a^{\prime}\right.$, $\left.\mathrm{a}+\mathrm{Da}^{\prime} \ldots \mathrm{a}+\mathrm{DN}-1 \mathrm{a}^{\prime}\right\}$, where $\mathrm{a}, \mathrm{a}^{\prime}$ represent a preferred pair of $\mathrm{m}$-sequences each having a period $\mathrm{N}=2 \mathrm{n}-1$, and $\mathrm{D}$ is a delay element.

\subsection{Kasami Sequences}


Kasami sequences generate a binary sequence with very small cross correlation value. Kasami sequence clasify as large and small set of sequence.the large set contain all the sequence of small set. Small set kasami sequence[3] [18] generates $2 n / 2$ no of binary sequences of length $N=2 n-1$. A shift register is used to generate a PN sequence with ' $n$ ' no of flipflops, where $n$ is even and positive number. For any $N$ length of PN sequence, $\mathrm{m} 1$ is first generated. By decimating $\mathrm{m} 1$ by $2 \mathrm{n} / 2+1$ number of bits, another PN sequence $\mathrm{m} 2$ of period $2 \mathrm{n} / 2-1$ has been genrated. By performing modulo- 2 addition between the bits of the sequence $\mathrm{m} 1$ and those of $\mathrm{m} 2$ and including the orginal PN sequence $\mathrm{m} 1$ in the above sequence set, altogther $2 \mathrm{n} / 2$ number of binary sequences has been produced.

\subsection{Minimum Correlation Spreading Code}

A minimum correlation spreading code is a code that minimizes average magnitude of auto correlation values except zero time shift and minimizes the average value of cross correlation [11] simultaneously. For minimum auto correlation, let's assume we have a spreading code, $\mathrm{s}$, of length $\mathrm{N}$, which can be represented as $[\mathrm{X} 1, \mathrm{X} 2 \ldots \mathrm{XN}-1, \mathrm{XN}] \mathrm{T}$, where $\mathrm{T}$ is the transpose operator. It is required to obtained the following for minimum autocorellation

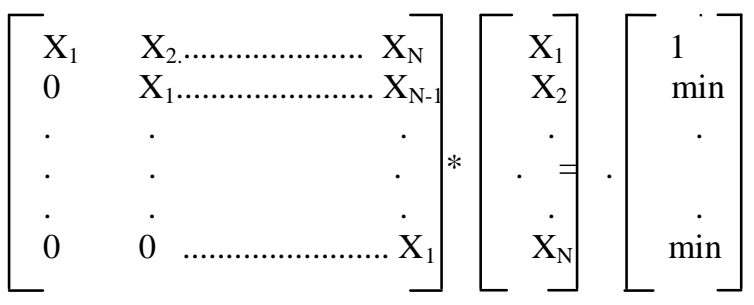

This is equivalent to minimization of function

$$
\begin{aligned}
& G_{I}=\mathbf{s}^{\mathbf{T}} . \mathbf{E} \cdot \mathbf{s}, \\
& \text { such that } \mathbf{s}^{\mathbf{T}} . \mathbf{s}=\mathbf{1} \text {. }
\end{aligned}
$$

Here, the eigenvector of a symmetric and rank 1 matrix, Ra gives minimum autocorelation spreading code in the following relation $\mathrm{Ra}=\mathrm{E}$, where $\mathrm{E}$ is a $(\mathrm{N} \times \mathrm{N})$ matrix with all element one.

In minimum cross correlation code the objective is to minimize the cross correlation values with a known spreading code. For this let us assume a spreading code, $\mathrm{x}$, of length $\mathrm{N}$, then find a code $\mathrm{s}$ that minimize the average magnitude of cross correlation. Where $\mathrm{x}$ and $\mathrm{s}$ can be represented as [X1 X2 $[\mathrm{Y} 1, \mathrm{Y} 2 \ldots \ldots \ldots \ldots \ldots \ldots . . . . . . \mathrm{YN}] \mathrm{T}$, respectively. For minimum cross-correlation code it is required to obtain

$$
\left[\begin{array}{ccc}
\mathrm{X}_{1} & \mathrm{X}_{2} \ldots \ldots \ldots \ldots \ldots \ldots \mathrm{X}_{\mathrm{N}} \\
0 & \mathrm{X}_{1} \ldots \ldots \ldots \ldots \ldots \ldots \mathrm{X}_{\mathrm{N}-1} \ldots \ldots \ldots \\
\cdot & \cdot & \cdot \\
\cdot & \cdot & \cdot \\
0 & 0 & \ldots \ldots \ldots \ldots \ldots \ldots \mathrm{X}_{1}
\end{array}\right] *\left[\begin{array}{c}
\mathrm{Y}_{1} \\
\mathrm{Y}_{2} \\
\cdot \\
\cdot \\
\cdot \\
\mathrm{Y}_{\mathrm{N}}
\end{array}\right]=\left[\begin{array}{c}
\min _{1} \\
\min _{2} \\
\cdot \\
\cdot \\
\cdot \\
\min _{\mathrm{N}}
\end{array}\right]
$$

To achive minimum cross correlation equation 6 can be formulated by minimizing the function

$$
G_{2}=\left(\mathbf{x}^{\mathrm{T}} \cdot \mathbf{E} \cdot \mathbf{s}\right)^{\mathrm{T}} \cdot\left(\mathbf{x}^{\mathrm{T}} \cdot \mathbf{E} \cdot \mathbf{s}\right),
$$


such that $\mathbf{s}^{\mathbf{T}} . \mathbf{s}=1$.

In this case the eigenvector of a symmetric and rank 1 matrix Rc gives minimum autocorelation spreading code in the following relation Rc=E.x.xT.ET.

In minimum correlation spreading we explore the idea of merging both Minimum Auto Correlation Code and Minimum Cross Correlation Code problems into a single problem. So we represent our optimization problem as

Minimize $G_{l}=\mathbf{s}^{\mathbf{T}}$. E. $\mathbf{S}$

Minimize $G_{2}=\left(\mathbf{x}^{\mathbf{T}} \cdot \mathbf{E} \cdot \mathbf{s}\right)^{\mathbf{T}} \cdot\left(\mathbf{x}^{\mathbf{T}} \cdot \mathbf{E} \cdot \mathbf{s}\right)$

such that $\mathbf{s}^{\mathbf{T}} . \mathbf{s}=1$

this can be written by a single crirerion minimization function using convex optimization as:

Minimize $G=K \cdot \mathbf{s}^{\mathbf{T}} \cdot \mathbf{E} \cdot \mathbf{s}+(1-K) \cdot\left(\mathbf{x}^{\mathbf{T}} \cdot \mathbf{E} \cdot \mathbf{s}\right)^{\mathbf{T}} \cdot\left(\mathbf{x}^{\mathbf{T}} \cdot \mathbf{E} \cdot \mathbf{s}\right)$

such that $\mathbf{s}^{\mathbf{T}} . \mathbf{s}=1$

where $0 \leq K \leq 1$

Finally $\mathbf{R}=K \times \mathbf{E}+(1-K) \times\left(\mathbf{E} \cdot \mathbf{x} \cdot \mathbf{x}^{\mathbf{T}} \cdot \mathbf{E}^{\mathbf{T}}\right)$ produces eigenvector of a symmetric and rank 1 matrix for minimum corelation spreading code.

Here, $\mathbf{R}$ generates $N-1$ number of eigenvector of zero eigenvalues which are orthogonal to each other.

\section{Proposed Algorithm}

The proposed algorithm for generation of orthogonal code for CDMA system has been described below:

STEP 1:Take any row of Walsh matrix $\mathrm{W}_{\mathrm{N}}$ as spreading code of length $N$ except all zeros

STEP 2:Create a matrix $\mathrm{X}$ of size $(\mathrm{N} \times \mathrm{N})$, whose first column is generated from the above step and all other columns are found by shifting the previous column by 1 bit position

STEP 3:Create a matrix $\mathrm{E}$ of size $(\mathrm{N} \times \mathrm{N})$ by taking all the elements equal to 1

STEP 4:Multiply $\mathrm{E}$ with each column vector of $\mathrm{X}$ and obtain a column vector of size $(\mathrm{N} \times 1)$. Thus $\mathrm{N}$ number of $(\mathrm{N} \times 1)$ column vectors are generated. Arrange all column vectors to generate a matrix A of size $(\mathrm{N} \times \mathrm{N})$.

Generate the matrix $\mathrm{B}$ where $\mathrm{B}=$ Transpose $(\mathrm{A})$

STEP 5:Evaluate $\mathrm{Ri}=\mathrm{K} . \mathrm{ET}+(1-\mathrm{K})$. Aci. Bri, Where, $\mathrm{K}$ is scalar quantity between 0 and 1 , ci and ri are ith column and ith row of matrices $\mathrm{A}$ and $\mathrm{B}$ respectively. $\mathrm{i}$ takes the value from 1 to $\mathrm{N}$. Thus $\mathrm{N}$ number of $\mathrm{N} \times \mathrm{N}$ symmetric matrices is generated

STEP 6:Generate eigenvalues and corresponding eigenvectors of $\mathrm{Ri}$

STEP 7:For each matrix $\mathrm{Ri}$, (N-1) number of zero eigenvalues and one non-zero eigenvalue have been generated. The corresponding eigenvectors of $(\mathrm{N}-1)$ number of zero eigenvalues are mutually orthogonal to each other

STEP 8: Finally, for $\mathrm{N}$ length code, $\mathrm{N} \times(\mathrm{N}-1)$ number of orthogonal codes are generated

\section{Simulation Results}

In this section, the simulation results are presented to compare performance of proposed OMCSC with the existing codes in terms of number of a number of factors, like code generation, correlation values and Bit Error 
Rate (BER) performances. For OMCSC code, $\mathrm{N}$ numbers of $\mathrm{N} \times \mathrm{N}$ matrices have been generated. Next, the eigenvalues associated with these matrices have been found out. It has been observed that $\mathrm{N}-1$ no. of zero eigenvalues and one nonzero eigenvalue exist for each $\mathrm{N} \times \mathrm{N}$ matrix. In this way, for all $\mathrm{N} \times \mathrm{N}$ matrices, $\mathrm{N} \times(\mathrm{N}-$ 1) number of zero eigenvalues and $N$ number of nonzero eigenvalues are evaluated. The eigenvector associated with these zero eigenvalues are found to be unique and orthogonal. To select the proper value of " $K$ " in the minimization function as described by equation (9), the magnitude of minimization function has been plotted with respect to the value of " $\mathrm{K}$ " and presented in Fig. 1. It is evident that the optimum value of " $\mathrm{K}$ " is 0.20 at which the minimization function attains its minimum value i.e. both the magnitudes of auto correlation and cross correlation become minimum.

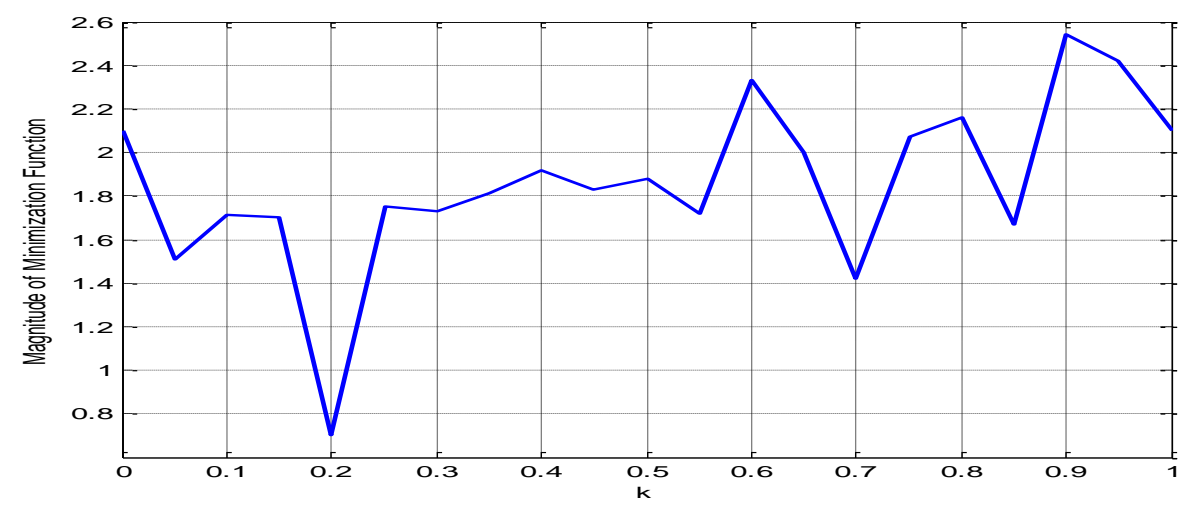

Fig.1 Plot of Minimization Function vs. K for code length of 16

\subsection{Number of Codes}

The proposed OMCSC algorithm generates large number of codes in comparison with existing codes like Walsh code and orthogonal Kasami code. For a given code of length $\mathrm{N}$, proposed code generates $\mathrm{N} \times(\mathrm{N}-1)$ number of distinct codes whereas Walsh code gives $\mathrm{N}$ and orthogonal small set Kasami code give $\operatorname{sqrt}(\mathrm{N}) \times(\mathrm{N}-1)$ number of distinct code. The detail comparison of proposed code and existing codes is shown in Table 1. From this it is clear that the proposed orthogonal MCSC algorithm outperforms the other code generation algorithms by providing a large number of users in a CDMA system.

Table 1. No of code members of different orthogonal codes for a fixed length code.

\begin{tabular}{lllll}
\hline $\begin{array}{l}\text { Length of the } \\
\text { Code }\end{array}$ & \multicolumn{4}{l}{ Number of distinct code members } \\
\cline { 2 - 5 } & Walsh & Orthogonal Gold code & Orthogonal small set Kasami Orthogonal proposed MCCSC \\
\hline 4 & 4 & 12 & 6 & 12 \\
16 & 16 & 240 & 60 & 240 \\
64 & 64 & 4032 & 504 & 4032 \\
256 & 256 & 65280 & 4080 & 65280 \\
\hline
\end{tabular}

\subsection{Correlation Property}

The average magnitudes of cross-correlation of the proposed code along with other existing codes have been presented in Fig.2. It is observed that the proposed spreading code gives zero cross correlation value at zero 
time shift which is an indication of maintaining orthogonality by the code. In contrast, other codes offer quite high magnitude of cross-correlation as compared to the proposed code. Hence it can be claimed that the proposed code can handle the effect of MAI in a better way than the other codes.

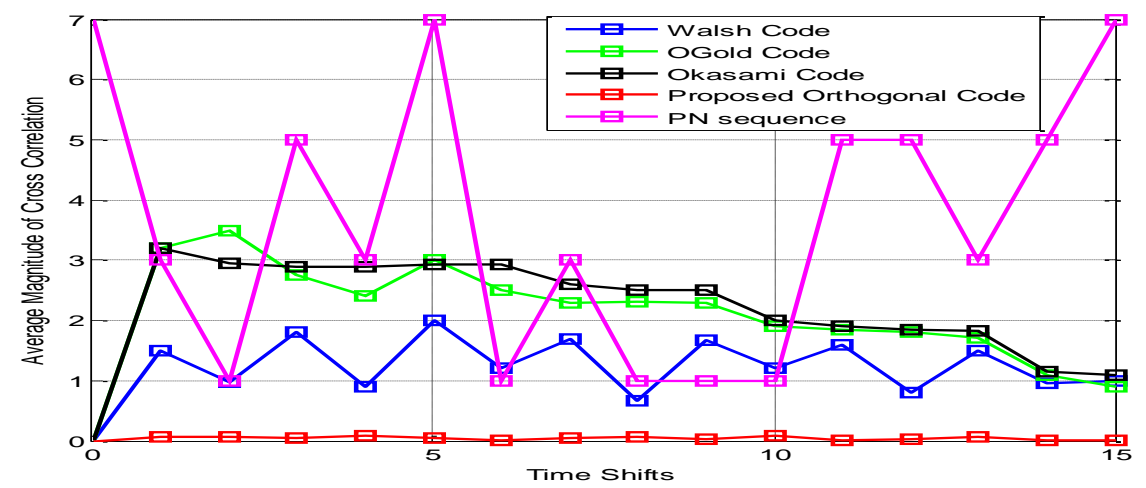

Fig.2 Comparison Based on Average Magnitude of Cross-correlation

The average magnitude of auto correlation of proposed OMCSC and existing codes are shown in Fig. 3. For this purpose, the average magnitude of auto correlation has been normalized to 1 in all the cases. From this Fig., it has been observed that our proposed code offers impulsive peak at zero time shift. Moreover, the average magnitude of the side lobes is low as compared to others. It is obvious from Fig. 3 that, except PN sequence, other existing codes are much inferior to the proposed code.

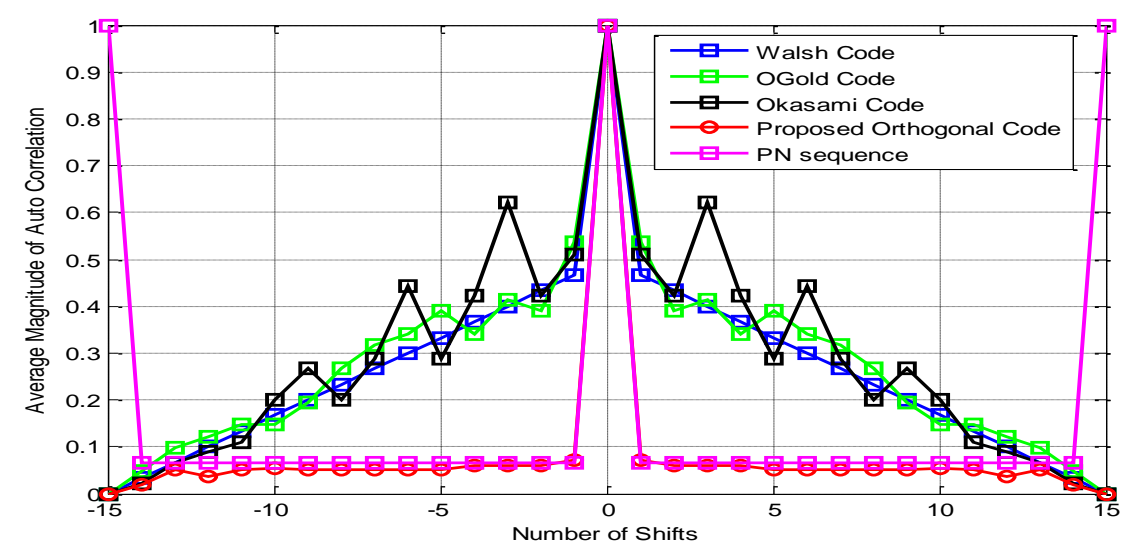

Fig.3 Comparison Based on Average Magnitude of Auto-correlation.

The ratio of peak autocorrelation to other shift autocorrelation $(\alpha)$ has been demonstrated in Table 2 . and the corresponding simulation result has been illustrated in Fig. 4. Likewise, the ratio of cross correlation to autocorrelation $(\beta)$ has been presented in Table 3.with the respective simulation result in Fig. 5.

A high value of $\alpha$ and a low value of $\beta$ is favourable for a good spreading code. The proposed OMSSC code possesses a high value of $\alpha$ as depicted in table 2 and Fig. 4 which consequently minimises the effect of ISI and thus reduces the chances of false synchronization at the receiver. Also, it has a low value of $\beta$ as illustrated in Table 3 and Fig.5. This minimum value of $\beta$ suggests that the proposed code can handle the effect of MAI in a 
better way than the existing spreading codes.

Table 2. Ratio of peak autocorrelation to other shift autocorrelation $(\alpha)$

\begin{tabular}{|c|c|c|c|c|c|c|c|c|c|c|c|c|c|c|c|}
\hline \multirow[t]{2}{*}{$\begin{array}{c}\text { Name of } \\
\text { code }\end{array}$} & \multicolumn{15}{|c|}{$\begin{array}{l}\text { Ratio of peak autocorrelation to other shift autocorrelation } \\
\text { shifts } \rightarrow\end{array}$} \\
\hline & 0 & 1 & 2 & 3 & 4 & 5 & 6 & 7 & 8 & 9 & 10 & 11 & 12 & 13 & 14 \\
\hline Walsh & 1 & 2.14 & 2.30 & 2.50 & 2.72 & 3.00 & 3.33 & 3.74 & 4.28 & 5.00 & 5.99 & 7.50 & 10.00 & 14.99 & 13.03 \\
\hline OKasami & 1 & 1.95 & 2.36 & 1.60 & 2.36 & 3.46 & 2.25 & 3.46 & 5.00 & 3.75 & 5.00 & 9.00 & 11.24 & 14.99 & 45.05 \\
\hline OGold & 1 & 1.86 & 2.56 & 2.41 & 2.92 & 2.56 & 2.92 & 3.1 & 3.72 & 5.12 & 6.83 & 6.83 & 8.19 & 10.24 & 20.49 \\
\hline PN Seq. & 1 & 15.00 & 15.00 & 15 & 15 & 15 & 15 & 15 & 15 & 15 & 15 & 15 & 15 & 15 & 15 \\
\hline $\begin{array}{l}\text { Purposed } \\
\text { code }\end{array}$ & 1 & 14.08 & 16.39 & 16.39 & 16.39 & 19.53 & 19.53 & 19.53 & 19.53 & 19.53 & 18.65 & 19.53 & 28.01 & 19.53 & 55.86 \\
\hline
\end{tabular}

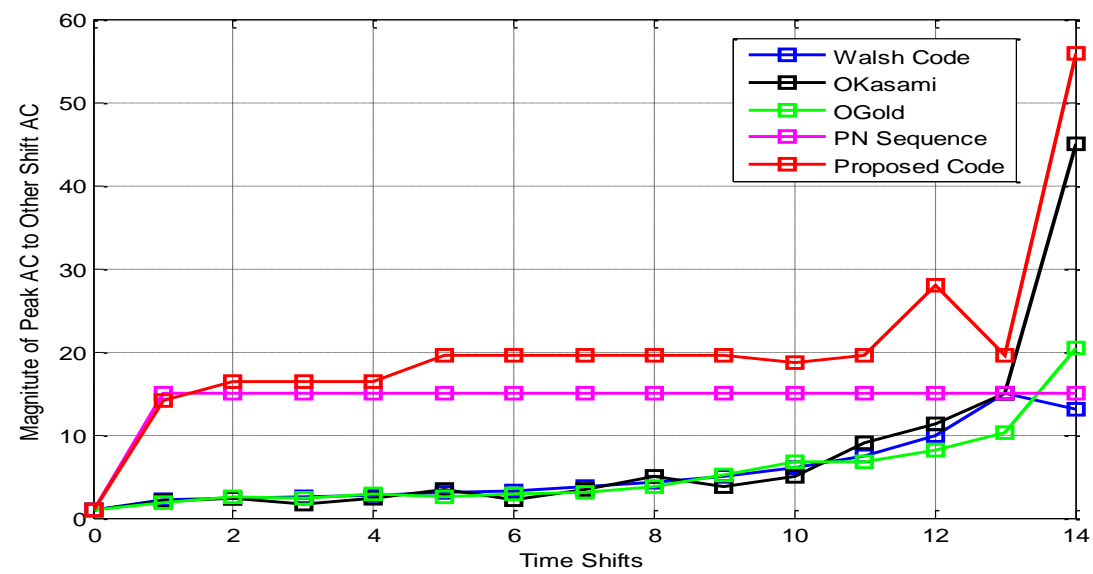

Fig.4 Comparison Based on Ratio of peak autocorrelation to other shift autocorrelation.

Table 3. Ratio of auto-correlation to cross-correlation $(\beta)$

\begin{tabular}{|c|c|c|c|c|c|c|c|c|c|c|c|c|c|c|c|}
\hline \multirow[t]{2}{*}{$\begin{array}{l}\text { Name of } \\
\text { the code }\end{array}$} & \multicolumn{15}{|c|}{$\begin{array}{l}\text { Ratio of Cross-correlation to Auto-correlation }(\beta) \\
\text { shifts } \rightarrow\end{array}$} \\
\hline & 0 & 1 & 2 & 3 & 4 & 5 & 6 & 7 & 8 & 9 & 10 & 11 & 12 & 13 & 14 \\
\hline Walsh & 0 & 3.21 & 2.23 & 4.50 & 2.45 & 6.00 & 4 & 6.37 & 2.87 & 8.40 & 7.19 & 12.00 & 8.00 & 22.89 & 28.52 \\
\hline OKasami & 0 & 6.21 & 6.98 & 4.66 & 6.86 & 10.10 & 6.57 & 8.99 & 12.50 & 9.37 & 10.00 & 17.10 & 20.87 & 27.45 & 52.21 \\
\hline OGold & 0 & 5.96 & 8.96 & 6.63 & 7.02 & 7.68 & 7.32 & 7.25 & 8.64 & 11.78 & 12.98 & 12.64 & 14.75 & 17.63 & 22.54 \\
\hline PN Seq. & 0.4 & 3 & 1 & 5 & 3 & 7 & 1 & 3 & 1 & 1 & 1 & 5 & 5 & 3 & 5 \\
\hline $\begin{array}{l}\text { Purposed } \\
\text { code }\end{array}$ & 0 & 0.98 & 1.05 & 0.92 & 1.33 & 0.95 & 0.01 & 0.85 & 1.21 & 0.49 & 1.62 & 0.25 & 0.85 & 1.50 & 0.73 \\
\hline
\end{tabular}




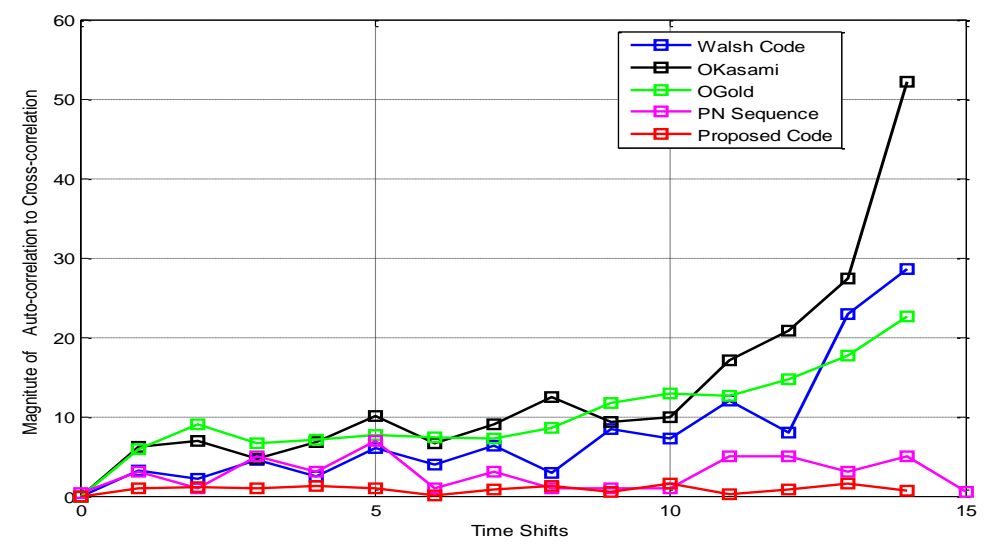

Fig.5 Comparison Based on Ratio Cross- correlation to Auto-correlation.

\subsection{Bit Error Rate Performance}

BER performance evaluation of proposed OMCSC has been carried out using Additive White Gaussian Noise (AWGN) channel under multiuser scenario. For this purpose, SIMULINK-based downlink CDMA system model has been used and the BER value of such system was also measured. One such model for twouser is shown in Fig.6.

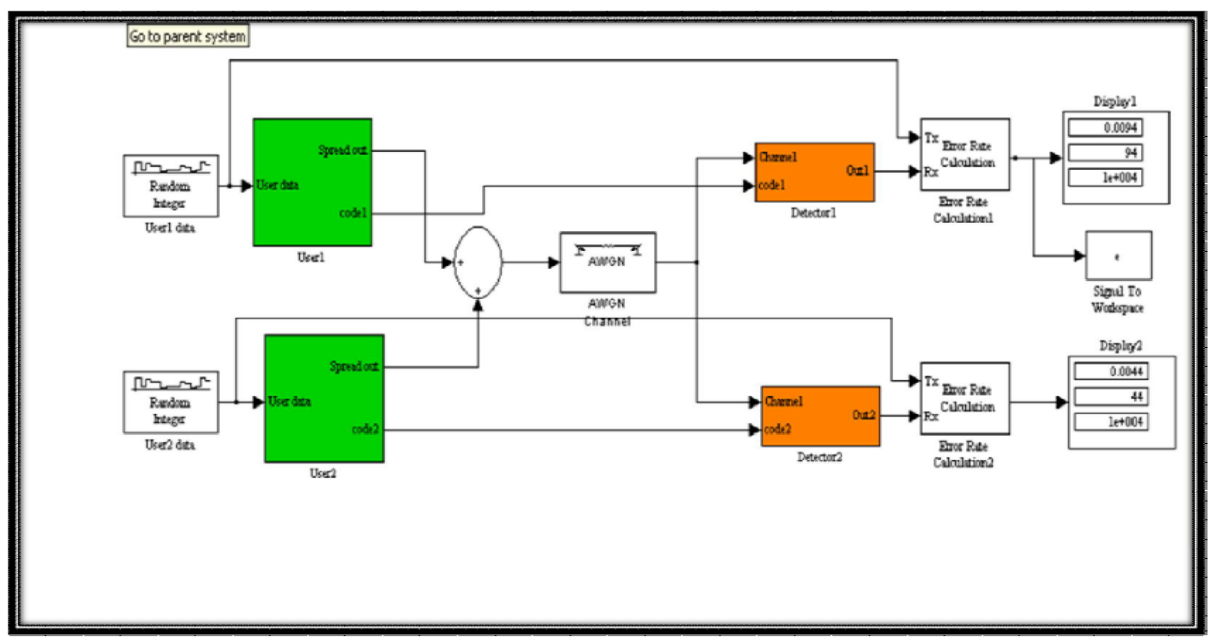

Fig.6. Simulation model of CDMA based downlink system for two-user scenario.

The SNR verses BER plots have been shown in Fig. 7, 8 and 9 for 8, 10 and 12 users respectively. For the purpose of comparison, the BER performances of three existing spreading codes have also been included in this study. 




Fig.7 Comparison of BER performances for synchronous downlink communication under 8 user scenario in AWGN channel

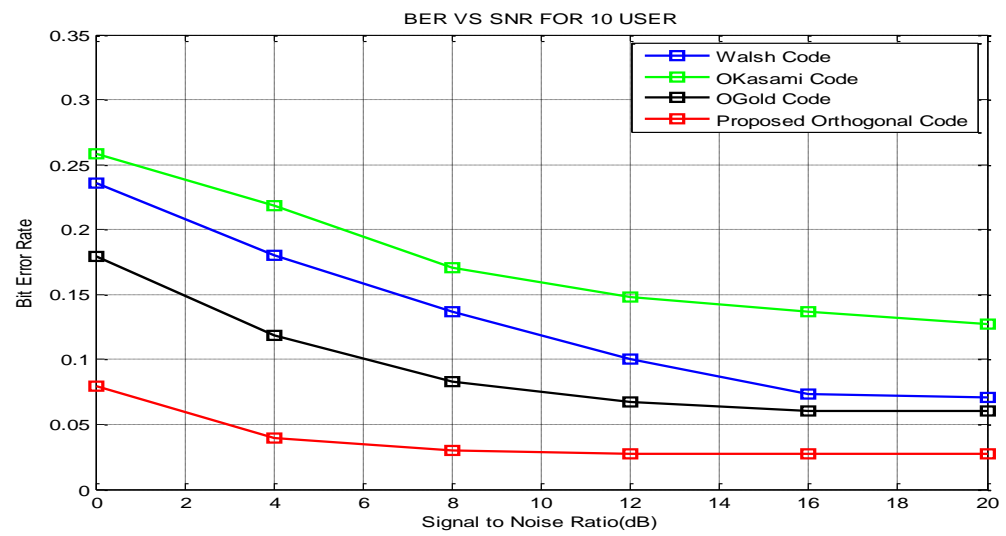

Fig.8 Comparison of BER performances for synchronous downlink communication under 10 user scenario in AWGN channel

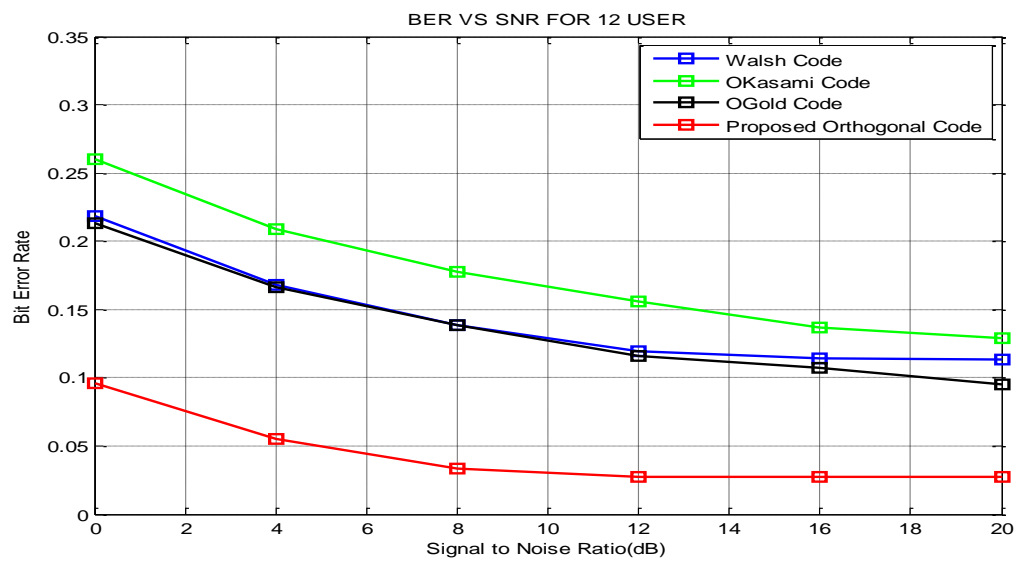

Fig.9 Comparison of BER performances for synchronous downlink communication under 12 user scenario in AWGN channel 
Fig. 10 and 11 describe the effect of number of users on BER performance of synchronous multiuser CDMA system using proposed OMCSC and existing codes for $12 \mathrm{~dB}$ and $20 \mathrm{~dB}$ SNR value respectively.

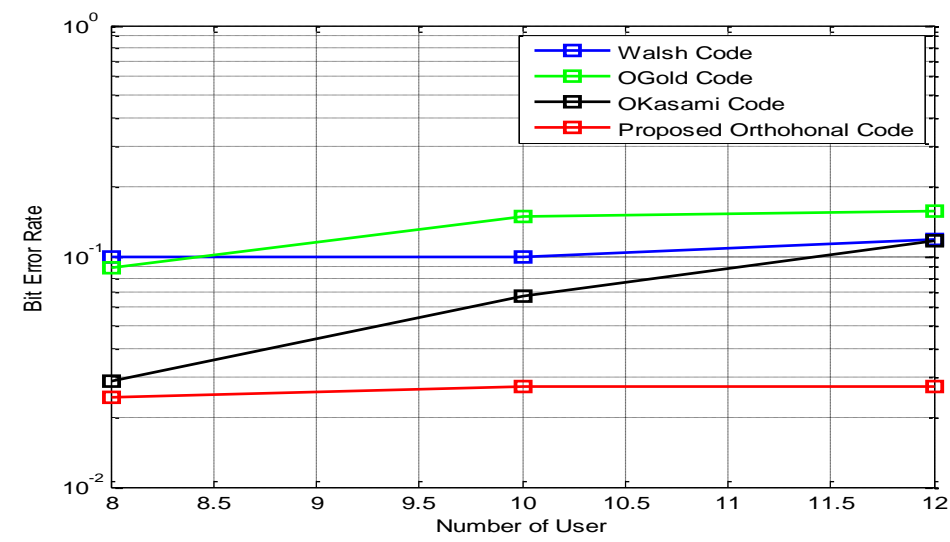

Fig.10 Multiuser BER performance for synchronous downlink communication under AWGN channel at SNR $=12 \mathrm{~dB}$

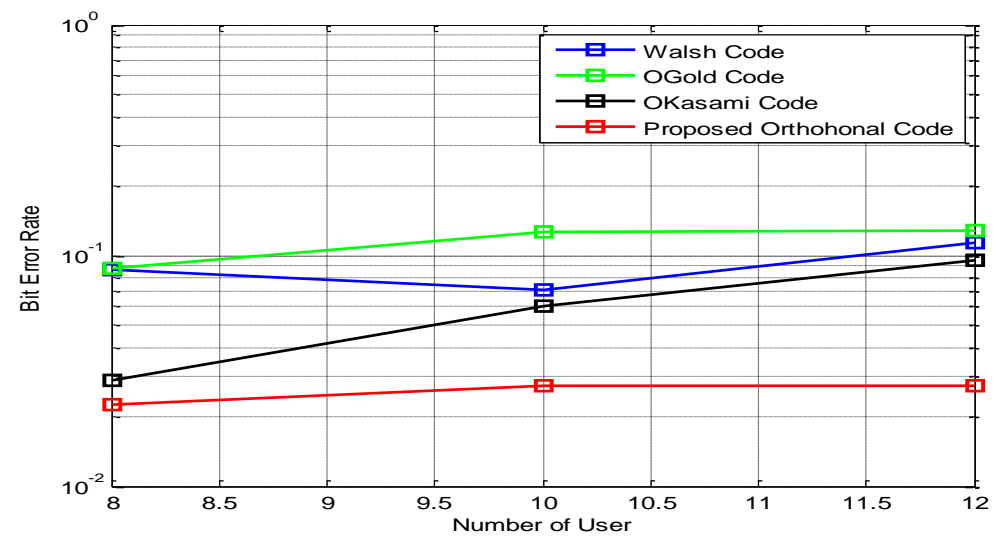

Fig.11 Multiuser BER performance for synchronous downlink communication under AWGN channel at SNR $=20 \mathrm{~dB}$

Table 4 and table 5 also illustrates the BER performances for different users of various spreading code like Walsh, Orthogonal Kasami, Orthogonal Gold and OMCSC code.

Table 4. BER Performances at 12dB for Different User

\begin{tabular}{|c|c|c|c|c|}
\hline & Walsh code & Orthogonal Kasami & Orthogonal Gold & OMCSC Code \\
\hline No. of user & BER & BER & BER & BER \\
\hline 8 & 0.0997 & 0.0289 & 0.0887 & 0.0449 \\
\hline 10 & 0.1000 & 0.0672 & 0.1482 & 0.0273 \\
\hline 12 & 0.1191 & 0.1162 & 0.1561 & 0.0271 \\
\hline
\end{tabular}


Table 5. BER Performances at 20dB for Different User

\begin{tabular}{ccccc}
\hline \multirow{2}{*}{ No. of user } & $\begin{array}{c}\text { Walsh code } \\
\text { BER }\end{array}$ & $\begin{array}{c}\text { Orthogonal Kasami } \\
\text { BER }\end{array}$ & $\begin{array}{c}\text { Orthogonal Gold } \\
\text { BER }\end{array}$ & $\begin{array}{c}\text { OMCSC Code } \\
\text { BER }\end{array}$ \\
\hline 8 & 0.0871 & 0.0286 & 0.0884 & 0.0227 \\
10 & 0.0712 & 0.0602 & 0.1273 & 0.0273 \\
12 & 0.1136 & 0.0953 & 0.1286 & 0.0271 \\
\hline
\end{tabular}

A close inspection of the above Fig. and tables reveals the fact that the proposed orthogonal code offers a lower value of BER irrespective of the number of users. Whereas, the degradation in the performance of the other codes is considerable with the increase in number of users. Hence the proposed code also outperforms the others in this respect.

\section{Conclusion}

In a CDMA system availability of more number of codes, minimum magnitude of cross correlation, impulsive peak auto correlation and BER performance plays a major role. So Code designer have to generate spreading code such that it support more number of user, good auto-correlation property, good BER performance with better cross-correlation property which eliminate undesirable effect like ISI, MAI by reducing the chance of false synchronisation. This paper present one algorithm to generate "a novel orthogonal minimum correlation spreading code" which offer a large number of codes in comparison to other existing code like Walsh code and OKasami code with better auto and cross correlation property. Also the BER performance of proposed OMCSC is suitable in AWGN channel with multiuser scenario. Hence we concluded that purposed OMCSC outperform other existing code and give one optimum solution for future CDMA communication system.

\section{Acknowledgements}

This work has been carried out under the guidance of Prof. Sudipta Chattopadhyay, Jadavpur University, Kolkota. Many thanks to Dr. D. Nageswar Rao, Vice-chancellor of CUTM and Dr. A.K Mishra, Registrar of CUTM, for providing and encouraging to carry out this work efficiently inside the campus. It's my pleasure to thank Professor P.K Dakua of ECE Dept. of CUTM for giving valuable suggestions in formatting the paper.

\section{References}

[1] Dixon R.C. (1976). Spread spectrum systems, John Wiley \& Sons, Inc.; New York.

[2] AJ. Viterbi. (1 995). CDMA Principles of Spread Spectrum Communication, Addison-Wesley.

[3] Esmael H. Dinan \& Bijan Jabbari, (Sep. 1998)." Spreading codes for direct sequence CDMA and wideband CDMA cellular networks", IEEE Communication Magazine.

[4] Moshavi, S. (1996). Multi-user detection for DS-CDMA communications. IEEE Communications Magazine, 34(10), 124-136.

[5] Sarwate, D. V., \& Pursley, M. B. (1980). Crosscorrelation properties of pseudorandom and related sequences. Proceedings of the IEEE, 68(5), 593-619.

[6] Jianxin,B., Yingmin, W., Hongxin, T., \& Kechu, Y. (2000). A new method to design CDMA spreading sequences. International Conference on Communication Technology Proceedings, WCC - ICCT 2000(Vol. 1 , pp. 526-529). 
[7] Develi, I. (2004), Detection of optimal spreading codes for DS-CDMA wireless systems with dispreading sequences weighted by adjustable chip waveforms, Science Direct Journal, vol. 342, no. 1, pp. 69-84.

[8] Wu, Z., \& Nassar, C. R. (2002). Novel orthogonal codes for DS-CDMA with improved cross correlation characteristics in multipath fading channels. The 5th International Symposium on Wireless Personal Multimedia Communications (Vol. 3, pp. 1128-1132).

[9] Amayreh, A. I., \& Farraj, A. K. (2007). Minimum autocorrelation spreading codes. Wireless Personal Communications, 40(1), 107-115.

[10] Farraj, A. K., \& Amayreh, A. I. (2009). Minimum cross correlation spreading codes. Wireless Personal Communications Journal, 48(3), 385-394.

[11] Farraj, A. K. (2010) Minimum correlation spreading codes design. Wireless Personal Communications Journal, 55, 395-405.

[12] H. Donelan and T.O' Farrell, "Methods for generating sets of orthogonal sequences", Electronics Letters, vol. 35, no. 18, pp. 1537-1538, September 1999.

[13] A. Chandra and S. Chattopadhyay, "Small set orthogonal Kasami codes for CDMA system", in Proc. Computers and Devices for Communication (CODEC-2009), Kolkata, December 2009.

[14] A.N. Akansu and R. Poluri, " Walsh-like nonlinear phase orthogonal codes for direct sequence CDMA communications", IEEE Transactions on Signal Processing, vol. 55, no.7, pp. 3800-3806, July, 2007.

[15] P. Fan and M. Darnell, Sequence Design for Communication Applications, U.K.: Research Studies Press, 1996.

[16] R. Gold, "Optimal Binary Sequences for Spread Spectrum Multiplexing," /€€€ Trans. Info. Theory, vol. IT-B, Oct. 1967, pp. 619-21.

[17] R. Gold, "Maximal Recursive Sequences with 3-Valued Recursive Cross-Correlation Functions," /€E€ Trans. Info. Theory, vol. IT-4, Jan. 1968, pp. 154-56.

[18] T. Kasami, "Weight Distribution Formula for Some Class of Cyclic Codes," Coordinated Science Lab., Univ. IL, Urbana. Tech. Rep., R-285, Apr. 1966.

[19] S.K.Mitra, Digital Signal Processing: A Computer Based Approach, McGraw Hill, 2001.

[20] Proakis, J. G., and Manolakis, D. G. (1996), Digital Signal Processing: Principles, Algorithms, and Applications, 3rd Edition, Prentice Hall.

\section{Author(s) Profile}

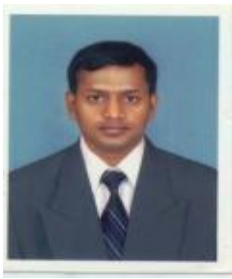

Mr.Shibashis Pradhan received his Master Degree in Electronics and Telecommunication Engineering from Jadavpur University, Kolkata in 2013. He is working as Assistant Professor in Centurion University in Department of ECE. Currently he is working in the area of Spreading code for CDMA system, an emerging domain that lies at the intersection of wireless communication and coding theory. Specifically, his research focuses on generation of spreading code for CDMA systems to solve various problems like Inter Symbol Interference (ISI) and Multiple Access Interference (MAI) in communication system.

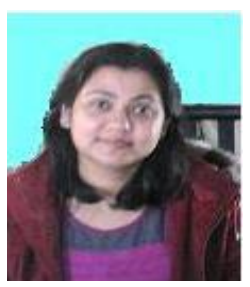

Sudipta Chattopadhyay received her B. Tech in Instrumentation Engineering in 1994 from Calcutta University, Kolkata- 700 009, India and M.E.Tel.E in 2001 from Jadavpur University, Kolkata - 700 032, India. She received her Ph.D from Jadavpur University, Kolkata - 700 032, India in 2012. She was a Lecturer in the Department of Electronics and Communication Engineering at Institute of Technical Education and Research, Bhubaneswar,India, from 1996-2001 and also worked as Lecturer, Sr. Lecturer and Asst. Professor in the Department of Electronics and Communication Engineering at Netaji Subash Engineering College, Kolkata - 
700 152, India, from 2001-2006. She is now working as an Assistant Professor in the Department of Electronics and Telecommunication Engineering, Jadavpur University, Kolkata - 700 032, India since 2006. She has published a number of papers in International/National Conferences. Her current research interests include Digital/Mobile Communication, Coding Theory and Digital Signal Processing. Mrs. Chattopadhyay is a member of IEEE since last 13 years. She is also an Executive Committee member of the Affinity Group - Women in Engineering $\|$ under IEEE Calcutta Section.

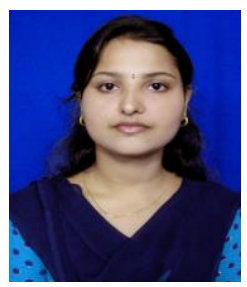

Ms.Sujatarani Raut received her M.Sc in Electronics Science and M.Tech in Electronics and Telecommunication Engg. From BU and BPUT respectively. Currently she is working as a lecturer in PN autonomous college khurda.

How to cite this paper: Shibashis Pradhan, Sudipta Chattopadhyay, Sujatarani Raut,"A Novel Orthogonal Minimum Correlation Spreading Code in CDMA System", IJWMT, vol.4, no.2, pp.38-52, 2014.DOI: 10.5815/ijwmt.2014.02.05 